IJPSR (2011), Vol. 2, Issue 5 (Research Article)
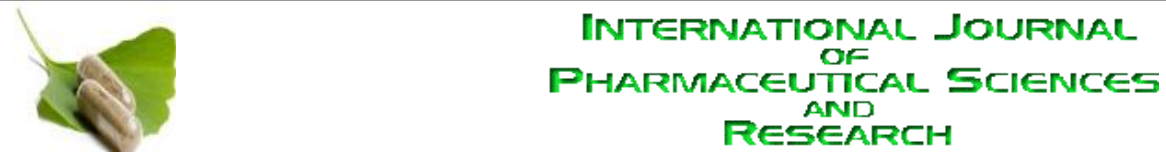

Received on 11 January, 2011; received in revised form 18 March, 2011; accepted 18 April, 2011

\title{
ANIMAL MODELS OF STRESS
}

Nitish Bhatia* ${ }^{1}$, Partha Pratim Maiti ${ }^{1}$, Abhinit Choudhary ${ }^{1}$, Atul Tuli ${ }^{1}$, Daljit Masih ${ }^{1}$, Mohd. Masih Uzzaman Khan ${ }^{1}$, Tasneem Ara $^{2}$ and Amteshwar Singh Jaggi ${ }^{3}$

Sri Sai College of Pharmacy ${ }^{1}$, Badhani, Pathankot, Panjab, India

Drug Testing Laboratory ${ }^{2}$, Dalgate, Srinagar, Jammu and Kashmir, India

Department of Pharmaceutical Sciences and Drug Research, Punjabi University ${ }^{3}$, Patiala, Panjab, India

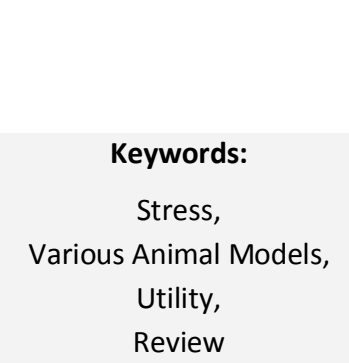

Correspondence to Author:

Nitish Bhatia

DISM, Assistant Professor, Sri Sai College of Pharmacy, Badhani, Pathankot, Panjab, India

\begin{abstract}
Stress undoubtedly has become an integral part of human life. Stressful conditions have a derogative effect on normal physiological functions leading to a variety of disease states. Many of the most prevalent diseases of the modern era like hypertension, diabetes, behavioral disorders, etc. have been implicated as one of the many ill-effects of chronic stress. Experimental models are required to better understand the progression of the disease and elaborate new therapy. Therefore a need to develop animal models of stress has always been realized. An ideal model should be able to reproduce each of the aspects of stress response and should be able to mimic the natural progression of the disease. Unfortunately different models of stress that have been used are able to evaluate some of the many biochemical or physiological parameters that get altered as a response to stress but are unable to fully mimic the pathophysiological changes caused by stress.
\end{abstract}


INTRODUCTION; Stress has become an integral part of human life and organisms are constantly subjected to stressful stimuli that affect numerous physiological processes. Stressful events can activate the Hypothalamo-Pituitary-Adrenal (HPA) axis ${ }^{1}$ and increase the release of Corticotrophin Releasing Hormone (CRH) from the hypothalamic paraventricular nucleus, causing the secretion of Adrenocorticotropin (ACTH) from anterior pituitary, which in turn stimulates the secretion of glucocorticoids from the adrenal cortex ${ }^{2}, 3$. Glucocorticoids possess broad spectrum of actions affecting expression and regulation of genes throughout the body readying the organism for changes in energy and metabolism required for coping ${ }^{4,5}$. Stress has been postulated to be involved in the etiopathogenesis of a variety of disease states including hypertension, coronary heart disease ${ }^{6,}$ gastric ulcers ${ }^{7}$, diabetes ${ }^{8}$, immunosuppression ${ }^{9}$, mental depression, memory loss ${ }^{10}$, and host of other diseases. The resultant disturbances may vary depending upon type, intensity, and the duration of a particular stressor and the strain $\backslash$ sex differentiation of the subjects ${ }^{11}$.

Different animal models for stress have been developed and used frequently to evaluate the antistress activity of compounds of both natural and synthetic origin. Research associated with stress has focused on identification, quantification, and characterization of the injured tissue for evaluation of different therapeutic modalities and understanding the mechanism of stress response. A requirement of all studies on stress is an adequate and appropriate animal model of stress. An ideal animal model should be able to reproduce each of the aspects of stress response and should be able to mimic the natural progression of the disease.

However, none of the models available is able to entirely reproduce stress response. Some models reproduce physical stress and associated ${ }^{\bullet}$ neuroendocrine changes ${ }^{12}$, whereas others better reproduce the psychological stress and associated behavioral changes ${ }^{13}$. Acute models do not reproduce the neuroendocrine dysfunction whereas a chronic model might be able to do so. Therefore, a correct model should be used to evaluate specific aspects of the stress response. Each model has inherent limitations including lack of stability, lack of predictability of tissue damage, and lack of adjustability. And hence a literature survey of more than 35 years (1970-2007) was conducted based on the description of the models, potential utilization of the models, and value of the models for testing of new medical interventions for the management of stress. The purpose of this review was to assess different models of stress.

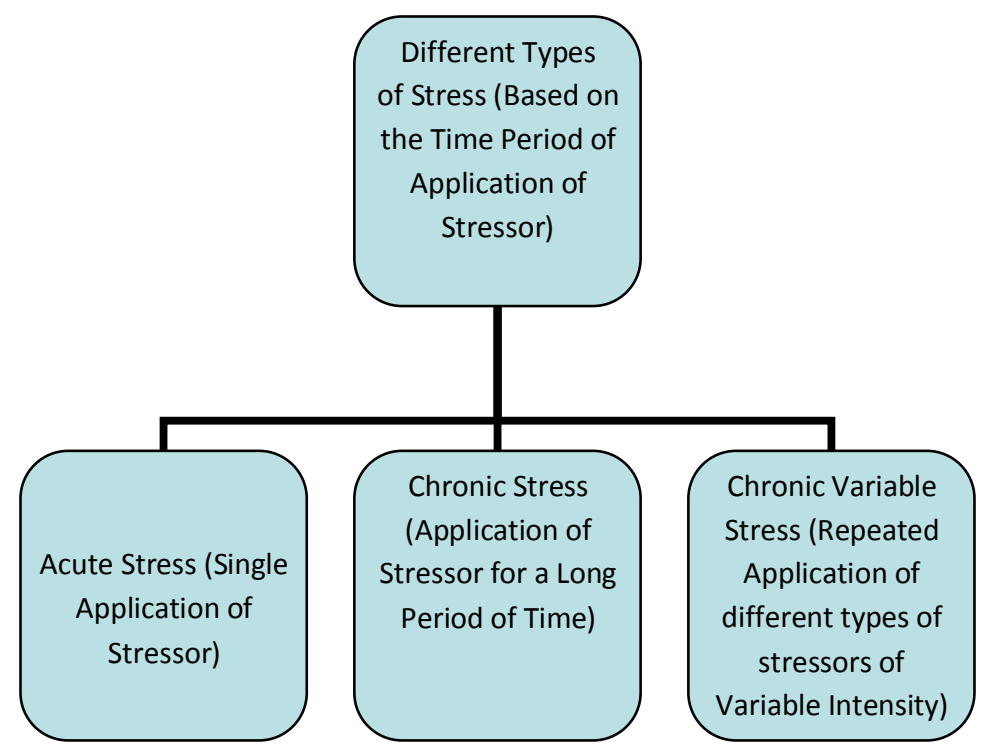

Animal Models commonly used:

Physical stress models: Animal models of stress that use physical stress can be subdivided into

- Temperature fluctuation induced stress;

1. Immersion in cold water with no escape

2. Cold environment isolation

Immobilization induced stress;

- Electric foot shock induced stress

- Forced swimming induced stress 
Psychological stress models: Animal models of stress that use psychological stress can be subdivided into:

- Neonatal isolation induced stress

- Predatory stress

- Day-night light change induced stress

- Noise induced stress.

Chronic unpredictable stress: Different stressors of mild to moderate intensity are applied on variable basis so as to prevent the emergence of adaptation or resistance to one particular type of stressor. It involves the use of both physical and psychological stress models in a random way.

\section{PHYSICAL STRESS MODELS:}

Temperature fluctuation induced stress: Acute change in temperature leads to stressful conditions by activation of temperature regulatory centre in the hypothalamus and subsequently HPA axis. It leads to acute release of adrenocortical hormones in the blood stream responsible for acute stressful response ${ }^{14}$. A sharp decrease in temperature using either cold water or freezer has been used frequently to induce acute stress.

1. Immersion in Cold Water (ICW): In this method, the rats are placed individually in a tank of cold water (depth $=15.5 \mathrm{~cm}$; temperature $=15-20^{\circ} \mathrm{C}$ ) where they either swim or remain in an upright position, keeping their heads above water level 15, 16, 17, 18 . This situation lasts for 15 minutes unless the rats sink. In that event, rats are removed before the cutoff time and are not included in the experiments. For acute stress, rats are sacrificed 30 minutes after the stress exposure. For chronic stress, animals are exposed to this stressor for 7-10 days. Rats are sacrificed $1 \mathrm{hr}$ after the last stress session. The major advantage of this type of stressor is that acute stress can be achieved in a relatively short period of time. However the major drawback of this model is that the body adapts to change in temperature on chronic exposure to low temperature and hence stress response gets highly diminished $^{19,20}$.

2. Cold environment isolation: In this method, rats are individually kept in a freezer with a temperature maintained at $4^{\circ} \mathrm{C}$. The rats are kept for 15 minutes once for acute stress and for 7-10 days to develop chronic stress ${ }^{21}$. This sharp fall in temperature leads to a sharp increase in the level of adrenocorticoids as explained above culminating in the development of stress response ${ }^{22,1}$. Unlike the ICW model, rats are prevented from drowning in cold water hence it is relatively safe model however it also suffers from same drawback of development of resistance/adaptation on chronic exposure.

Immobilization induced stress: Immobilization has been used extensively as a stressor for the study of stress-related biological, biochemical and physiological responses in animals ${ }^{12,23,24}$. Immobilization can be produced in two different ways. Animal can be either kept immobilized in a semi cylindrical acrylic tube (4.5 $\mathrm{cm}$ diameter and $12 \mathrm{~cm}$ long) with proper holes in it for air to pass ${ }^{25}$. Another way is to keep the animal with its limbs stretched on a board and its limbs are immobilized with adhesive tape. Movement of head is restricted by keeping the head in a metal loop coiled around the neck. The rats are kept immobilized in either of the above two ways for 150 minutes once to produce acute stress and for 7-10 days to produce chronic stress ${ }^{26}$. The major advantage of using immobilization as a model of stress is that it produces an inescapable physical and mental stress to which adaptation is seldom exhibited ${ }^{23}$.

Electric foot shock induced stress: Electric foot shock (EFS) of mild intensity has also been used as a stressor. Rodents are very susceptible even to mild shock and exhibit rapid stress response. Researchers have used electric foot shock of varying degree to produce stressful conditions and hence to evaluate adaptogenic activity of various compounds. Stress by electric foot 
shock is given by placing the rats individually in a chamber with an electrified floor. Rats receive unavoidable electric foot shocks with an intensity of 3 $\mathrm{mA}, 200 \mathrm{~ms}$ of duration and a frequency of 1 per second over a 5 -min period. For acute stress response, the rats are exposed once and sacrificed after 15 minutes of stress.

Chronic stress is also produced by repeating the same treatment for 7-10 days and rats are sacrificed $1 \mathrm{~h}$ after the last stress session ${ }^{15}$. Some researchers have modified the method in which rats are subjected to inescapable electric foot shock for 60 minutes $(0.15$ $\mathrm{mA}$ shock, on a variable interval schedule with a mean inter shock interval of 60 seconds) ${ }^{27}$. The biggest advantage of this model is that it effectively produces high degree of stress in the animal. The major disadvantage of this model is the hazard of electric shock causing death of the animal and special caution that is required to perform this methodology.

Forced swimming induced stress: It is the tendency of the living being to escape or avoid a noxious stimuli/condition. If the animal is not able to escape the stressful stimuli or it feels threatened, the animal will show stress response. This principle is used for developing forced swimming model for inducing stress in laboratory animals. In order to produce swimming induced stress, rats are made to swim in a cylinder (30 cm diameter and filled to a height of $20 \mathrm{~cm}$ with 15 $\mathrm{cm}$ of space above the head of the rat) for a single session of $2 \mathrm{~h}$ duration for acute stress, or for one 2-h session a day for five consecutive days for chronic stress $^{28}$.

Some authors have used forced swimming in warm $\left(20^{\circ} \mathrm{C}\right)$ water for 3 minutes with the total session lasting for $1 \mathrm{~h}{ }^{29}$. Although forced swimming induced stress is a highly safe model, adaptation to chronic swimming induced stress has been reported and interstrain differences between rats to forced swimming behavior have also been documented ${ }^{30}$.

\section{PSYCHOLOGICAL STRESS MODELS:}

Neonatal isolation stress: Early life events have profound consequences on subsequent quality of life. It has been shown that the early life stress of neonatal isolation in rats has immediate and enduring neural and behavioral effects ${ }^{31}$. Such effects may reflect, in part, stress-induced morphological changes in hippocampus and other brain regions ${ }^{32}$. In fact, the hippocampus provides negative feedback regulation of the hypothalamic-pituitary-adrenal (HPA) axis ${ }^{33}$ and hence neonatal isolation induced stress can represent the stress response that may lead to neurodegenration at an early stage of life.

This stress procedure is also useful in evaluating the effect of stress on cognition and memory development. In the neonatal isolation procedure, the litter of the inbred strain is removed from the cage on second day after the birth, weighed and placed individually in an opaque plastic container $19 \mathrm{~cm}$ diameter and $8 \mathrm{~cm}$ deep) with no bedding for $1 \mathrm{~h}$ (between 09:00 and 12:00) in a heated $\left(30^{\circ} \mathrm{C}\right.$ ), humidity controlled chamber with white noise to mask other pups' calls.

The chamber has to be located in a room separate from animal colony facility. Containers are placed 20$30 \mathrm{~cm}$ apart. After $1 \mathrm{~h}$ period the litters are placed back with their dams in home cage ${ }^{34,35}$. This isolation procedure continues up to 8 days and hence it is used to induce chronic stress only. Neonatal isolation stress model has be used extensively to demonstrate the effect of early lifetime stress on vulnerability to addiction ${ }^{36}$, and response to psychostimulants by impairment of hippocampal-dependent context induced fear in adult male rats.

Predatory stress: Direct encounter of an animal with its natural predator is one of the most stressful and anxiogenic event it can face and it leads to rapid development of 'flight or fight' response ${ }^{37}$. Exposure of rodents to natural predators or to their odors may 
induce stress like states ${ }^{38}$. Under such circumstances, there is rapid sympathetic activation leading to rise in the levels of adrenocorticoids in blood causing acute stress response to develop. Direct encounter with a predator has been effectively used to evaluate the biochemical and physiological changes produced during such stressful conditions ${ }^{39}$. Predatory stress in mice is induced by series of short exposures to natural predator like cat ${ }^{40}$ or to any substance having the odor of cat like the fecal pellets of cat ${ }^{41}$.

In one of the methods, mice are placed individually in different cages and after four initial 20-min cage habituation sessions each subject is submitted to two randomly-assigned 20-min predator confrontation sessions. Change in behavioral pattern such as locomotion, shrieking like voices and endocrinological changes after the stress exposure are observed ${ }^{42}$. Another free-exploration test ${ }^{43}$ was used consists of a PVC box $(30 \times 20 \times 20 \mathrm{~cm})$ covered with Plexiglas and subdivided into six equal square exploratory units, which are all interconnected by small entries.

It could be divided in half lengthwise by closing three temporary partitions. Approximately $20 \mathrm{~h}$ before cat exposure, each subject is placed in one half of the apparatus with the temporary partitions in place, in order to be familiarized with it. The floor of this half was covered with fresh sawdust and the animal is given unlimited access to food and water. On the test day, mice of each strain are randomly allocated to the following four groups.

(a) Naive + clay: animals are exposed to both familiar and novel compartments by removal of the temporary partitions. The novel compartment contains three modeling odor-free clay pellets.

(b) Naive + feces: animals are exposed to both familiar and novel compartments. The novel compartment contains three cat feces pellets.

(c) Exposed + clay: subjects are removed from the freeexploration box and confronted individually with a cat during a 5-min session. The cat cage consists of a PVC box $(82 \times 56 \times 62 \mathrm{~cm})$ subdivided into two compartments, one containing the cat, the other the mouse. Separation consists of a transparent PVC wall with holes allowing the cat to reach the other side with its paws. The mouse is then put back in the freeexploration apparatus and is exposed $1 \mathrm{~h}$ later to both familiar and novel compartments. The novel compartment contains three modeling odor-free clay pellets.

(d) Exposed + feces: same as previous group, but the novel compartment contains three pellets of feces from the cat used during exposure. The behavior of the mouse is observed under red light for $5 \mathrm{~min}$ via a closed circuit TV camera by an observer located in an adjacent room.

The following parameters are recorded:

(a) Time spent in the novel compartment;

(b) Total unit entries and (c) total number of rearings.

The results are expressed as mean percentage of time spent in the novel compartment, mean total number of novel unit changes, and mean total number of rearings. Marmosets (Callithrix penicillata) have also been employed for induction of predatory stress in a test battery known as Marmoset Predator Confrontation Test (MPCT) ${ }^{44}$. This model compares the behavioral response of experienced versus naïve adult black tufted-ear marmosets in confrontation with a taxidermized wild-cat predator stimulus.

After four initial 20-min cage habituation sessions, each subject is submitted to two randomly-assigned 20-min predator confrontation sessions. Confrontation with the predator induces significant behavioral changes; i.e., proximic avoidance and tsik-tsik alarm call. Anti-stress drug administration, concomitant to predator exposure, reverses the behavioral changes observed ${ }^{45}$. Predator induced stress is an established model to induce short term acute stress response but 
its major disadvantage is development of habituation to predator exposure hence the use of this model for inducing stress is justified for developing only acute stress.

Day-night light change induced stress: Changes in the circadian rhythm have profound effect on physical and psychological well being of an individual ${ }^{46}$. Laboratory animals, when subjected to abrupt changes in daynight light pattern, exhibit acute stress response 32 . Changes in circadian rhythms are regulated by pineal gland through the secretion of melatonin ${ }^{47}$. Melatonin is released from the pineal gland in response to dark or dim light where as its functional antagonist serotonin is secreted in response to bright light. It is this serotoninmelatonin cycle that is responsible for regulation of sleep-awake state of the body ${ }^{48,49}$.

To induce stress, cages of rat or mice are kept under bright light from 19:00 h over night (in the dark phase) and cages are kept in dark room with no light from $12: 00 \mathrm{~h}$ in the light phase for 180 minutes for 7-10 days 13. This method is suitable for inducing short term stress response. Generation of stress can be evaluated by measuring the biochemical parameters associated with chronic stress response ${ }^{50}$. The major disadvantage of this model is that it can be effectively used to generate short term stress response as on repeated exposure to this type of stressor, the animal adapts to the changed day-night light pattern. This major drawback can be minimized by using this model as a part of chronic unpredictable stress protocol.

Noise induced stress: Noise as a stressful stimulus is a widely accepted fact. A large number of people are exposed to potentially hazardous levels of noise levels in daily modern life. Experimental studies have demonstrated ultra structural modifications in rat cardiomyocytes mainly in mitochondria due to noise stress. These subcellular alterations are related to an imbalance in calcium homeostasis, which is supposed to be sustained by increased catecholamine innervations ${ }^{51}$. When noise exposure of any kind exceeds $90 \mathrm{~dB}$, noise becomes a stressor ${ }^{52}$. Noise stress has a depletory effect on free radical scavenging enzymes in the brain leading to moderate to severe oxidative stress ${ }^{53}$ which can be a potential basis for hearing loss ${ }^{54}$. Noise stress in laboratory rats can be produced by loudspeakers ( $15 \mathrm{~W})$, driven by a white noise generator $(0-26 \mathrm{kHz})$, installed $30-\mathrm{cm}$ above the cage. Thus a noise level can be set at $100 \mathrm{~dB}$ or above uniformly throughout the cage and can be monitored by a sound level meter. Each animal to be treated is exposed to noise stress for $4 \mathrm{~h} /$ day for 15 days. Control group rats are also kept in the above described cage during the corresponding period of time, without noise stimulation to avoid the influence of handling stress on evaluation of effects due to noise exposure ${ }^{55,56}$. The effect of noise stress exposure can be determined by estimating the brain biogenic amine level.

Chronic Variable (Unpredictable) Stress Models: The major disadvantage of both physical stress models and psychological stress models is the development of adaptation/resistance on chronic exposure. The changes in physiological and behavioral responses to chronic stress can be related to the adaptation of the HPA axis. When the same stressor is repeated, the HPA response undergoes desensitization or become stable as it has been reported that rodents repeatedly exposed to restraint stress exhibited a habituated corticosterone response, when they were subsequently challenged with an acute exposure to restraint ${ }^{57,58}$.

On the other hand, the exposure to a multiple stress paradigm produced continued elevation in corticosterone levels, when the animals were subsequently subjected to acute restraint stress ${ }^{57}$. It has also been suggested that the adaptations of HPA axis depend on type, duration and severity of the stress regime ${ }^{58,42}$. To prevent the development of resistance, Chronic Unpredictable Stress (CUS) models have been developed which involve the use of various physical and psychological stressors in a predetermined manner so that the animal is not able 
to adapt to the stressor. Adaptation to one type of stressor has been effectively prevented by employing various stressors such as immobilization stressor for 15 minutes followed by overnight sleep deprivation and rotation of the cage at a predetermined speed (horizontal shakes at high speed) for 50 minutes followed by swim stress in water $\left(20^{\circ} \mathrm{C}\right)$ of 4 minutes ${ }^{59}$.

Wetting the saw dust bedding of the animal all day to restrict movement followed by electric foot shock (ten shocks of one second duration each, in an unpredictable manner, at the intensity level of 0.4-1.8 $\mathrm{mA}$ ) and stroboscopic light (for $13 \mathrm{~h}, 10 \mathrm{~Hz}$ ) has also been used as a part of CUS protocol ${ }^{60}$. Some researchers have used exposure to predator odor induced stress as a part of CUS protocol, in which mice are placed in a novel cage containing cat litter soiled with cat feces and urine ${ }^{61}$. Various authors have modified the stress models in order to accommodate them in their respective CUS protocol. Other additional stressors that have been applied as a part of CUS protocol are tail pinch with a clothes-pin placed $1 \mathrm{~cm}$ distal from the base of the tail for $5 \mathrm{~min}$, strong illumination during predicted dark phase for $12 \mathrm{~h}$, movement restriction in a small cage $(11 \mathrm{~cm} \times 16 \mathrm{~cm} \times$ $7 \mathrm{~cm}$ ) for $2 \mathrm{~h} \mathrm{59,}$, ether anaesthesia until loss of reflex 62 , and subcutaneous $0.9 \%$ saline injection.

Chronic variable stress models have been proven to be more useful as they are devoid of the problem of resistance in the animal species towards the commonly used stressors and also have the advantage of the development of effective and long-term stress response. Thus CUS models are nowadays the preferred models for generation of a stress response.

DISCUSSION: A wide variety of animal models for inducing stress are available which can be used to understand the pathogenesis of the disease and to evaluate new treatment modalities. Animal models have been instrumental in elucidating cellular and extra cellular mechanisms causing stress related pathological conditions. The exact mechanism of generation and progression of stress response is still unknown. Models available for inducing stress suffer a major drawback that they are not able to fully reproduce stress related physiological and biochemical changes simultaneously. Other major disadvantage of most of the models of stress is development of resistance on chronic application. As animal body has the inherent property to adapt to adverse condition, stress response to aversive stimuli diminishes with time. One of the methods to prevent is to use chronic unpredictable stress parameter which employs a battery of stressful stimuli to induce inadaptable stress response. Therefore the researcher can either choose the model that will best reproduce the aspect of stress under investigation or can switch over to multiple stress model paradigms to avoid adaptation to one type of stress.

\section{REFERENCES:}

1. Kvetnansky R., J. Jelokova, M. Rusnak, S. Dronjak, B. Serova, B. Nankova and E.L. Sabban: Novel stressors exaggerate tyrosine hydroxylase gene expression in the adrenal medulla of rats exposed to long-term cold stress, in: R. Kvetnansky (Ed.) Stress: Neural, Endocrine and Molecular studies, Taylor and Francis, London, Edition 1, 2002, 121-128.

2. Pacak K, Kvetnansky R, Palkovits M, Fukuhara K, Yadid G, Kopin IJ and Goldstein DS: Adrenalectomy augments In-vivo release of norepinephrine in the paraventricular nucleus during immobilization stress. Endocrinology 1993; 133: 1404-1410.

3. Venihaki M, Gravanis A and Margioris AN: Comparative study between normal rat chromaffin and $\mathrm{PC} 12$ rat pheochromocytoma cells: Production and effects of Corticotropin Releasing Hormone (CRH). Endocrinology 1997; 138: 698-704.

4. Akil HA and Morano MI: Stress In: Bloom FE and Kupfer DJ editors, Psychopharmacology: The fourth generation in progress. New York: Raven Press, 1995; pp. 773-785.

5. Levine $S$, Development determinants of sensitivity and resistance to stress. Psychoneuroendocrinology 2005; 30: 939-946.

6. Roy MP, Kirschbaum C and Steptoe A: Psychological cardiovascular and metabolic correlates of individual differences in cortisol stress recovery in young men. Psychoneuroendocrinology 2001; 26: 375-391.

7. Yadin E. and Thomas E: Stimulation of the lateral septum attenuates immobilization-induced stress ulcers. Physiology \& Behavior 1996; 59: 883-886.

8. Fitzpatrick F, Christedd N, Durant S, Dardenne M, Nunez EA and HomoDelarche F: Glucocorticoids in non obese diabetic (NOD) mouse Basal serum levels, effect of endocrine manipulation and immobilization stress. Life Sciences 1992; 50: 1063-1069. 
9. Purret SB: Quantitative aspects of stress-induced immunomodulation. International Journal of Immunology and Pharmacology 2001; 1: 507-520.

10. Gareri P, Falconi U, Fazio P and De Sarro G: Conventional and new antidepressant drugs in the elderly. Progress in Neurobiology 2000; 61: 353-396.

11. Kioukia-Fougia N, Antoniou K, Bekris S, Liapi C, Christofidis I and Papadopoulou-Diafoti Z: The effect of stress exposure on hypothalamic-pituitary-adrenal axis, thymus, Thyroid hormones and glucose levels. Progress in Neuropharmacology Biology and Psychiatry 2000; 26: 823-830.

12. Kvetnansky R. and Mikulai L: Adrenal and urinary catecholamines in rats during adaptation to repeated immobilization stress. Endocrinology 1970; 81: 738-743.

13. Marcelo TM, Fabio CC and Planeta CS: Chronic restraint or variable stresses differently affect the behavior, corticosterone secretion and body weight in rats. Physiology and Behavior 2007; 90: 29-35.

14. Sapolsky RM, Krey LC and McEwen BS: The neuroendocrinology of stress and aging: the glucocorticoid cascade hypothesis. Endocrinology Reviews 1986; 7: 284-301.

15. Retana-Marquez S, Bonilla-Jaime $H$, Vazquez-Palacios $G$, Dominguez-Salazar E, Martinez-Garcia R and Velazquez J: Body weight gain and diurnal differences of corticosterone changes in response to acute and chronic stress in rats. Psychoneuroendocrinology 2003; 28: 207-227.

16. Iwona BL, Konarzewski $M$ and Sodowski B: Effect of cold acclimation and repeated swimming on opioid and nonopoid swim stress-induced analgesia in selectively bred mice. Physiology \& Pharmacaology 2003; 78: 345-350.

17. Fernandez-Landiera J, Analysis of the cold-water restraint procedure in gastric ulceration and body temperature. Physiology \& Behaviour 2004; 82: 827-833.

18. Yun Tan, Qi Gan and Kneupfer MM: Central $\alpha$-adrenergic receptors and corticotrophin releasing factor mediate hemodynamic responses to acute cold stress. Brain Research 2003; 968: 122-129.

19. Pitman DL, Ottenweller JE and Natelson BH: Plasma corticosterone levels during repeated presentation of two intensities of restraint stress: Chronic stress and habituation. Physiology \& Behavior 1988; 43: 47-56.

20. Blustein JE, Ciccolone $L$ and Bresh PJ: Evidence that Adapatation to Cold Water Swim-Induced Analgesia Is a Learned Response. Physiology \& Behavior 1998; 63: 147-150.

21. Kvetnansky R, Gewitz GP, Weise VK and Kopin IJ: Catecholaminesynthesizing enzymes in the rat adrenal gland during exposure to cold. American Journal of Physiology 1971; 220: 928-931.

22. Staratakis CA and Chrousos GP: Neuroendocrinology and pathophysiology of the stress system. Annals of New York Academy of Sciences 1995; 771: 1-18.

23. Kasuga $\mathrm{S}$, Ushijima $\mathrm{M}$, Morihara N, Itakura $\mathrm{Y}$ and Nakata $\mathrm{Y}$ : Effect of Aged Garlic Extract (AGE) on hyperglycemia induced by immobilization stress in mice. Japanese Journal of Pharmacology 1999; 114:191-197.

24. Marty O, Martyn M and Gavalda A: Inhibition of corticosteroidbinding globulin caused by severe stressor is apparently mediated by the adrenal but not the glucocorticoid receptors. Endocrinology 1997; 6: 159-164.

25. Das A, Kapoor K, Sayeepriyadarshani AT, Dikshit M, Palit G and Nath C: Immobilization stress induced change in brain acetylcholinesterase activity and cognitive function in mice. Pharmacology Research 2000; 42: 213-217.

26. Dronjak S. and Gavrilovic L: Effect of stress on catecholamine stores in central and peripheral tissues of long term socially isolated rats. Brazilian Journal of Medical and Biological Research 2006; 39: 785-790.

27. Taysse L, Christin D, Delamanche S, Bellier B and Breton P: Peripheral ChE Inhibition Modulates Brain Monoamines Levels and $c$-fos Oncogene in mice subjected to a stress situation. Neurochemical Research 2005; 30(3): 391-402.

28. Kitchen I. and Pinker SR: Antagonism of swim-stress induced antinociception by the delt-opioid receptor antagonist naltrindole in adult and young rats 1990; 100 (4): 685-688.

29. Armario A, Gavalda A and Marti J: Comparison of the behavioral and endocrine response to forced swimming stress in five inbred strains of rats. Psychoneuroendocrinology 1995; 20 (8): 879-890.

30. Kuhn CM, Pauk J and Schanberg SM: Endocrine responses to mother-infant separation in developing rats. Developmental. Psychobiology 1990: 23: 395-410.

31. Kosten TA, Zhang XY and Kehoe P: Neurochemical and behavioral responses to cocaine in adult male rats with neonatal isolation experience. Journal of Pharmacology and Experimental Therapeutics 2005b; 314: 661-667.

32. Herman JP and Cullinan WE, Neurocircuitry of stress: Central control of the hypothalamic-pituitary-adrenocortical axis. Trends in Neurosciences 1997; 20: 78-84.

33. Kosten TA, Miserendino MJD and Kehoe P: Enhanced acquisition of cocaine self-administration in adult rats with neonatal isolation stress experience. Brain Research 2000; 875: 44-50.

34. Kosten TA, Sanchez H, Zhang XY and Kehoe P: Neonatal isolation enhances acquisition of cocaine self administration and food responding in female rats. Behavioural Brain Research 2004; 151: 137-149.

35. Kosten TA and Kehoe P: Neonatal isolation is a relevant model for studying the contributions of early life stress to vulnerability to drug abuse: Response to Marmendal et al. 2004. Developmental psychobiology 2005a; 47:108-10.

36. Lupien SJ, Ouelle-Morin I, Hupback A, Walker D, Tu MT and Buss C: Beyond the stress concept: Allostatic load-a developmental biological and cognitive perspective. In: D. Cicchetti (Ed.), Handbook series on developmental psychopathology. Taylor and Francis, Wisconsin 2006; pp. 784-809.

37. Adamec RE and Shallow T: Lasting effects of rodent anxiety of a single exposure to a cat. Physiology \& Behavior 1993; 54: 101-109.

38. Marilia B, Marco G, Anna A, Souto V, Gabriela V, Katarina S, Naia VB and Carlos T: Persistent anxiety like behavior in marmosets following a recent predatory stress condition: Reversal by diazepam. Pharmacology Biochemistry and Behavior 2007; 86(4): 705-711.

39. Blanchard RJ and Blanchard DC: Antipredator defensive behaviors in a visible burrow system. Journal of Comparative Physiology 1989; 103: 70-82. 
40. Berton F, Vogel E and Belzung C: Modulation of mice anxiety in response to cat odor as a consequence of predator's diet. Physiology \& Behavior 1998; 65: 247-254.

41. Blanchard RJ, Nikulina JN, Sakai RR, Mckittrick C, McEwen B and Blanchard C: Behavioral and endocrine change following chronic predatory stress. Physiology \& Behavior 1998: 63: 561-569.

42. Griebel G, Belzung C, Misslin R and Vogel E: The free-exploratory paradigm: An effective method for measuring neophobic behavior in mice and testing potential neophobia-reducing drugs. Behavioral Pharmacology 1993; 4: 637-644.

43. Cilia J and Piper DC: Marmoset conspecific confrontation: an ethologically-based model of anxiety. Pharmacology Biochemistry and Behavior 1997; .58: 85-91.

44. Barros M, Silva de Souza MA, Huston JP and Carlos T: Multibehavioral analysis of fear and anxiety before, during, and after experimentally induced predatory stress in Callithrix penicillata. Pharmacology Biochemistry and Behavior 2004; 78 : 357-367.

45. Atcheson JB and Tyler FH: Circadian rhythm: man and animals. In: Greep RO, Astwood EB. (Eds.), Handbook of Physiology, section 7, vol. VI. American Physiological Society, Washington, 1975; pp. 127-134.

46. Nicholson S, Lin JH, Mahmoud S, Campbell E, Gillham B and Jones $\mathrm{M}$ : Diurnal variations in responsiveness of the hypothalamopituitary-adrenocortical axis of the rat. Neuroendocrinology 1985; 40: 217-224.

47. Bermudez FF, Forbes JM and Injidi MH: Involvement of melatonin and thyroid hormones in the control of sleep, food intake and energy metabolism in the domestic fowl. Journal of Physiology 1983; 337: 19-27.

48. Hamm HE, Takahashi JS and Menaker M: Light-induced decrease of serotonin $\mathrm{N}$-acetyltransferase activity and melatonin in the chicken pineal gland and retina. Brain Research 1983; 266: 287293.

49. Rai D, Bhatia G, Sen T and Palit G: Comparative study of perturbations of peripheral markers in different stressors in rats. Canadian Journal of Physiology and Pharmacology 2003; 81: 11391146.

50. Paparelli A, Soldani P, Breschi MC, Martinotti E, Scatizzi R and Berrettini $S$ Effect of sub acute exposure to noise on the noradrenergic innervation of the cardiovascular system in young and aged rats: a morphofunctional study. Journal of Neural Transmission (General Section) 1992; 88: 105-113.
51. Ramsey JM: Modern stress and the disease process. In: Basic Physiology. California: Addison-Wesley Publishing Company 1982; pp 177-79.

52. Samson J, Devi S, Ravindran R and Senthivelan M: Effect of noise stress on free-radical scavenging enzymes in brain. Environmental Toxicology and Pharmacology 2005; 20: 142-148.

53. Fechter LD: Oxidative stress: a potential basis for potentiation of noise-induced hearing loss. Environmental Toxicology and Pharmacology 2005; 19: 543-546.

54. Ravindran R., Rathinaswamy SD, Samson J and Senthilvelan M: Noise-Stress-Induced Brain Neurotransmitter Changes and the Effect of Ocimum sanctum (Linn) Treatment in albino Rats. Journal of Pharmaceutical Sciences 2005; 98: 354-360.

55. Manikandan S and Devi SR Antioxidant property of $\alpha$-asarone against noise-stress-induced changes in different regions of rat brain. Pharmacological Research 2005; 52: 467-474.

56. Magarinos, AM and Mc Evans BS: Stress induced atrophy of apical dendrites of hippocampal CA3c neurons: comparison of stressors. Neuroscience 1995; 69: 83-88.

57. Gadek-Michalska A and Bugajski J: Repeated handling, restraint, or chronic crowding impairs the hypothalamic-pituitaryadrenocortical response to acute restraint stress. Journal of Physiology and Pharmacology; 54: 449-459.

58. Ortiz J, Fitizgerald LW, Lane S, Terwillinger R and Nestler EJ: Biochemical adaptations in the mesolimbic dopamine system in response to repeated stress. Neuropsychopharmacology 1996; 14: 443-452.

59. Margus K, Matrov D, Ko iv K, Eller M, To nissaar M and Harro J: Changes in regional long-term oxidative metabolism induced by partial serotonergic denervation and chronic variable stress in rat brain. Neurochemistry International 2007; 22: 223-227.

60. Anisman H, Prakash P, Merali Z and Poulter MO: Corticotropin releasing hormone alterations elicited by acute and chronic unpredictable stressor challenges in stressor-susceptible and resilient strains of mice. Behavioural Brain Research 2007; 181: 180-190.

61. Renard GM, Suarez MM, Levin GM and Rivarola MA: Sex differences in rats: Effect of chronic stress on sympathetic system and anxiety. Physiology and Behavior 2005; 85: 363-369.

62. Ladd CO, Thrivikraman KV, Huot RL and Plotsky PM: Differential neuroendocrine response to chronic variable stress in adult Long Evans rats exposed to handling-maternal separation as neonates. Psychoneuroendocrinology 2004; 30: 520-533. 